\title{
Extrasynaptic Vesicular Transmitter Release from the Somata of Substantia Nigra Neurons in Rat Midbrain Slices
}

\author{
Erica H. Jaffe, ${ }^{1,2}$ Alain Marty, ${ }^{2}$ Albert Schulte, ${ }^{1}$ and Robert H. Chow ${ }^{3}$ \\ ${ }^{1}$ Division of Molecular Biology of Neuronal Signaling, Max-Planck-Institut für Experimentelle Medizin, Göttingen, Germany \\ D-37075, 2Arbeitsgruppe Zelluläre Neurobiologie, Max-Planck-Institut für Biophysikalische Chemie, Göttingen, Germany \\ D-37077, and ${ }^{3}$ Membrane Biology Group and Department of Physiology, University of Edinburgh Medical School, \\ Edinburgh, United Kingdom EH8 9AG
}

\begin{abstract}
Substantia nigra neurons release dopamine from their somatodendritic regions. A long-unresolved question is whether this release occurs by exocytosis or by a nonvesicular mechanism. We used carbon fiber microelectrodes in a brainstem slice to assay secretion from single cell bodies that had been cleared of connective tissue. Amperometry at the carbon fiber microelectrodes revealed unitary events in $\sim 90 \%$ of cells in resting conditions. These events had charge integrals ranging from a few femtocoulombs to several hundred femtocoulombs (fC). Local glutamate application enhanced the event frequency by 3.5-fold on average and up to 10-fold in highly responsive cells, although the mean charge integral was not modified. Local application of a high $\mathrm{K}^{+}$-containing saline had effects similar to
\end{abstract}

Over the past two decades investigators have accumulated evidence that the classical view of neurotransmission-involving fast $\mathrm{Ca}^{2+}$-triggered fusion of transmitter-containing vesicles at well defined synapses-may not be valid in the substantia nigra. The cell bodies and short "basal" dendrites of these dopaminergic neurons reside in the pars compacta and extend axons to the neostriatum (the nigrostriatal tract). Longer dendrites are located in the pars reticulata. Surprisingly high concentrations of dopamine are found not only in the regions of the synaptic terminals in the neostriatum (where synaptic vesicles are located) but also in the dendrites (Björklund and Lindvall, 1975). This finding led to the suggestion that dopamine may be secreted not only at synapses in the neostriatum but also from dendrites (Björklund and Lindvall, 1975). Subsequent studies have confirmed the presence of $\mathrm{Ca}^{2+}$-dependent dopamine secretion in the substantia nigra (for review, see Cheramy et al., 1981). In addition, dopamine has been noted to have a local inhibitory action on the firing of substantia nigra neurons (for review, see Lacey, 1993). Despite

Received Sept. 15, 1997; revised Feb. 23, 1998; accepted March 2, 1998.

This work was supported by a Royal Society Research Grant (to R.H.C.) and the Deutsche Forschungsgemeinschaft (SFB 406), and by grants from the Deutsche Forschungsgemeinschaft (to E.J.), from the European Community Training and Mobility Program (to A.S.), and from the Howard Hughes Medical Institute (to R.H.C.). We gratefully acknowledge the kind support of Professor Walter Stühmer (Max Planck Institute for Experimental Medicine, Division of Molecular Biology of Neuronal Signaling).

Correspondence should be addressed to Dr. R. H. Chow, Membrane Biology Group, Department of Physiology, University of Edinburgh Medical School, Edinburgh, UK.

Dr. Jaffe's present address: Instituto Venezolano de Investigaciones Cientificas, Caracas, Venezuela.

Dr. Schulte's present address: Department of Physiology, University of Edinburgh Medical School, Edinburgh, UK.

Copyright (C) 1998 Society for Neuroscience $\quad 0270-6474 / 98 / 183548-06 \$ 05.00 / 0$ those of glutamate. The frequency of resting and stimulated amperometric events was much lower at $21-22^{\circ} \mathrm{C}$ than at $32-35^{\circ} \mathrm{C}$. The addition of $\mathrm{Cd}^{2+}(50 \mu \mathrm{M})$, a blocker of voltagedependent $\mathrm{Ca}^{2+}$ channels, to the bath solution blocked the stimulatory effects of glutamate. These results suggest that dopamine is released from the somata of substantia nigra neurons by exocytosis and that this mechanism is regulated by neuronal electrical activity. More generally, this study demonstrates the applicability of carbon fiber microelectrodes to the measurement of quantal monoamine secretion in brain slices.

Key words: substantia nigra; carbon fiber; exocytosis; dopamine; amperometry, pars compacta the findings of local dopamine release and action, electron micrograph studies have not revealed a significant number of vesicles in the pars compacta and reticulata (Wassef et al., 1981), raising the possibility of a nonvesicular mechanism of release.

Studies using the false neurotransmitter hydroxydopamine to localize dopamine (Mercer et al., 1979) and using gold-tagged antibody to label vesicular monoamine transporter-2 (VMAT-2, the vesicular monoamine transporter that is found in neurons) (Nirenberg et al., 1996) have shown that dopamine and VMAT-2 are found in membrane-delimited compartments both in the somata and in the dendrites of substantia nigra neurons. These membrane-delimited compartments include clear vesicles of small size, large dense-core vesicles, tubulovesicular structures tentatively assigned to the smooth endoplasmic reticulum, and the trans-Golgi network. Thus, although early electron microscopy studies did not find many synaptic vesicles (Wassef et al., 1981), substantia nigra neurons do contain membranous organelles that could be the vehicles of exocytosis.

In this paper we performed amperometric measurements with carbon fiber microelectrodes to examine the mechanism of dopamine release from the somata of substantia nigra neurons. Amperometry is a highly sensitive electrochemical technique based on recording the redox current arising from released oxidizable or reducible transmitters. When applied to isolated, cultured neuroendocrine and neuronal cells, it can detect the quantal (vesicular) release of catecholamine transmitters (Wightman et al., 1991; Urena et al., 1994; Chen et al., 1995; Zhou and Misler, 1995; Chow et al., 1996). In slices of substantia nigra, related voltammetric methods have been used to monitor increases in the concentration of dopamine in the solution superfusing the slices (Rice et al., 1994; Cragg et al., 1997). However, electrochemical 
detection of neuroamine secretion in slice preparations at the single-cell level has not proven feasible until now.

\section{MATERIALS AND METHODS}

Slice preparation. Rats $14-16$ d old were decapitated under Metofane anesthesia, and the brain was removed. A block of midbrain containing the substantia nigra was cut out and fixed on a vibratome. During cutting, slices were bathed in an ice-cold incubation solution containing (in $\mathrm{mM}$ ): $\mathrm{NaCl} 125, \mathrm{KCl} 2.5, \mathrm{NaH}_{2} \mathrm{P}_{4} 1.25, \mathrm{NaHCO}_{3} 26, \mathrm{CaCl}_{2} 1, \mathrm{MgCl}_{2} 4$, ascorbate 0.5 , and glucose 10 and gassed with $95 \% \mathrm{O}_{2} / 5 \% \mathrm{CO}_{2}$. Coronal slices $200 \mu \mathrm{m}$ thick containing the caudal substantia nigra and the accessory optic tract were cut and incubated at $32^{\circ} \mathrm{C}$ for $1 \mathrm{hr}$ in the above solution. Slices then were transferred to a recording chamber where they were visualized with a $40 \times$ water immersion objective and superfused ( $1.5 \times$ the bath volume/min) with a solution containing (in $\mathrm{mm}$ ): $\mathrm{NaCl}$ $125, \mathrm{KCl} 2.5, \mathrm{NaH}_{2} \mathrm{PO}_{4} 1.25, \mathrm{NaHCO}_{3} 26, \mathrm{CaCl}_{2} 2, \mathrm{MgCl}_{2} 1$, and glucose 10 and gassed with $95 \% \mathrm{O}_{2} / 5 \% \mathrm{CO}_{2}$. Except where otherwise indicated, experiments were conducted at $32-34^{\circ} \mathrm{C}$.

Carbon fiber microelectrode fabrication. The carbon fibers (PAN T650, 5-6 $\mu \mathrm{m}$ diameter; Amoco Performance Products, Greenville, SC) first were heated at $390^{\circ} \mathrm{C}$ for $3 \mathrm{hr}$ to remove sizing compound from the surface of the carbon. These fibers were used to manufacture two types of carbon fiber microelectrodes of comparable sensitivity and noise. One type was prepared with polyethylene insulation (Zhou and Misler, 1995; Chow et al., 1996). The other type used an insulation method involving electrodeposition of an insulating organic "paint" (EDP; Canguard, BASF Lacke und Farben AG, Muenster, Germany) (Schulte and Chow, 1996). A $3 \mathrm{~cm}$ length of PAN T650 carbon fiber was attached to a $5 \mathrm{~cm}$ length of 0.5 -mm-diameter copper wire with a small drop of conductive carbon paste. Then the carbon fiber, attached to the copper wire, was threaded into a patch pipette that previously had been pulled on a standard vertical patch-pipette puller to yield a tip having a diameter of $\sim 10 \mu \mathrm{m}$, until the carbon fiber passed out of the tip and the copper wire lodged at the narrowed neck of the pipette tip. A small drop of twocomponent glue was applied to the back end of the pipette to fix the copper wire firmly in the pipette, and the glue was allowed to harden for several hours. SYLGARD (Rhône-Poulenc Rorer, France) was applied to the tip region to hold the carbon fiber in place and to provide a watertight seal. An electrical connection to a DC power supply was made to the copper wire extending from the back of the patch pipette. Electrodeposition of the insulating paint was performed by applying a constant voltage of 3-5 V for 2-4 min between the carbon fiber dipped into EDP and a platinum counterelectrode. Coated carbon fibers were heatcured at $195^{\circ} \mathrm{C}$ for $2 \mathrm{~min}$. To enable electrical connections, we soldered a gold-plated pin to the copper wire at the back of the electrode. The tip of the carbon fiber was cut with a scalpel blade under a dissection microscope before each experiment.

Although both electrode types were used and both were able to detect the quantal events from substantia nigra neurons, the EDP-insulated electrodes were more convenient, because the tips were straighter and passed more readily beneath the microscope water immersion objective.

Testing of microelectrode properties. Before the experiments, all carbon fiber microelectrodes were tested for noise and sensitivity. Their electrical noise was measured with the freshly cut tip of the electrode dipped into the standard saline solution with a $\mathrm{Ag} / \mathrm{AgCl}$ reference electrode. Only electrodes having noise lower than $200 \mathrm{fA}$ root mean square at 1 $\mathrm{kHz}$ were used. The electrodes also were tested for peak reduction currents in cyclic voltammograms in a solution containing $1 \mathrm{~mm}$ $\mathrm{K}_{3} \mathrm{Fe}(\mathrm{CN})_{6}$ and $500 \mathrm{~mm} \mathrm{KCl}, \mathrm{pH} 3.0$. A voltage ramp from +500 to -500 $\mathrm{mV}$ and then back to $+500 \mathrm{mV}$ was applied at $100 \mathrm{mV} / \mathrm{sec}$ between the carbon fiber and a $\mathrm{Ag} / \mathrm{AgCl}$ reference electrode. The cyclic voltammograms routinely displayed the expected sigmoidal shape (see Fig. 1), with peak currents in the range of 800 to $1000 \mathrm{pA}$-near the value predicted for microelectrodes having an electroactive diameter of $\sim 5 \mu \mathrm{m}$ (Schulte and Chow, 1996). In the rare case of an electrode not having a peak current within this range, the tip was recut.

Cleaning procedure. The mediocaudal region of the pars compacta, near the accessory olfactory tract, was used in the present experiments. A very large proportion ( $>85 \%)$ of the neurons in this structure has been shown to be dopaminergic (Yung et al., 1991). The neurons were large, closely packed together, and had somata that were either spindle-like or pyramidal (Yung et al., 1991). For recording, we selected neurons located well within the pars compacta on the basis of (1) healthy appearance (smooth membranes and nonvacuolated), (2) superficial localization within the slice, and (3) presentation of a smooth somatic surface without emerging dendrites. The experimental chamber was rotated on the microscope stage until the edge of the membrane area to be recorded from was perpendicular to the axis of the cleaning pipette. Cleaning pipettes had tip diameters of $8-10 \mu \mathrm{m}$ and were filled with standard bath saline. They were mounted on the same micromanipulator that served subsequently to hold the carbon fiber microelectrode. Cleaning involved alternating positive and negative pressure (Edwards et al., 1989). Only the selected side of the neuron was freed of covering tissue, to minimize the risk of cell damage. Recordings were initiated as quickly as possible after cleaning, after the cleaning pipette had been replaced with the carbon fiber microelectrode (see Fig. 1A).

Amperometric recording. A carbon fiber microelectrode was mounted onto the headstage of the patch-clamp amplifier. The microelectrode tip then was touched gently to a cell, as illustrated in Figure $1 \mathrm{~A}$, and current signals were recorded under voltage clamp at a holding potential of 720 or $800 \mathrm{mV}$. Sampling was at $5 \mathrm{kHz}$ with low-pass filtering of $1 \mathrm{kHz}$. Traces shown in the figures additionally have been filtered at $200 \mathrm{~Hz}$.

The cell was stimulated by pressure-ejecting solution from a glass pipette (tip opening $\sim 3 \mu \mathrm{m}$ ) positioned in the vicinity of the recorded neuron. Control experiments showed that the observed amperometric responses could not be ascribed to movements of the carbon fibers because of solution flow, nor could they be attributed to extracellular recordings of action potentials, which would have been biphasic or principally downward deflections instead of the uniformly upward inflections that were recorded.

\section{RESULTS}

\section{Spontaneous amperometric events}

For this work we used neurons located in the mediocaudal region of the substantia nigra pars compacta. This region contains a particularly high density of tyrosine hydroxylase immunoreactive cells and yields a correspondingly high level of dopamine release in bulk voltammetry experiments (Yung et al., 1991; Cragg et al., 1997). In our first attempts to record amperometric signals from substantia nigra cells, we positioned carbon fiber microelectrode tips at the soma or dendrite of the cells, but we were unable to measure convincing amperometric events. Touching the carbon fiber microelectrode tip to the brain slice tissue led to a dramatic decrease (by factors as large as 10 or more) in voltammetric currents measured in solutions of test agents (Fig. 1B), suggesting that the lack of successful recordings was attributable to the reduction in the sensitivity of electrodes because of contact with extracellular materials in the brain slices. We therefore decided that, before recording from a substantia nigra neuron, we would dissect away the tissue overlying the somata, using a "cleaning pipette" (Edwards et al., 1989).

Just after a cell was cleaned, a freshly cut carbon fiber tip was positioned in the recording chamber $\sim 50 \mu \mathrm{m}$ above the cell, and a 1-2 min control recording was taken. Then the fiber was pushed gently into contact with the cell membrane, and a "cell contact" recording of 2 min was taken. Figure $1 A$ illustrates the appearance of a substantia nigra neuron after the cleaning procedure and the position of a carbon fiber during a recording. In many instances spontaneous amperometric events with variable shapes were observed in the cell contact recording, as illustrated in Figure $2 A$. Rarely, there were larger, more irregularly shaped events, such as the one shown in the second row of Figure $2 A$. These may represent multiples of the underlying quanta and/or another type of vesicle.

Over a total of 27 cells that were assayed in control conditions from 14 preparations, 25 had spontaneous events, a percentage of 93\%. The frequency of the spontaneous events was generally low, from 0.1 to 30 events/min, and in most cases it fell within the range of $0.3-3$ events/min (Fig. $2 B$ ). 
A

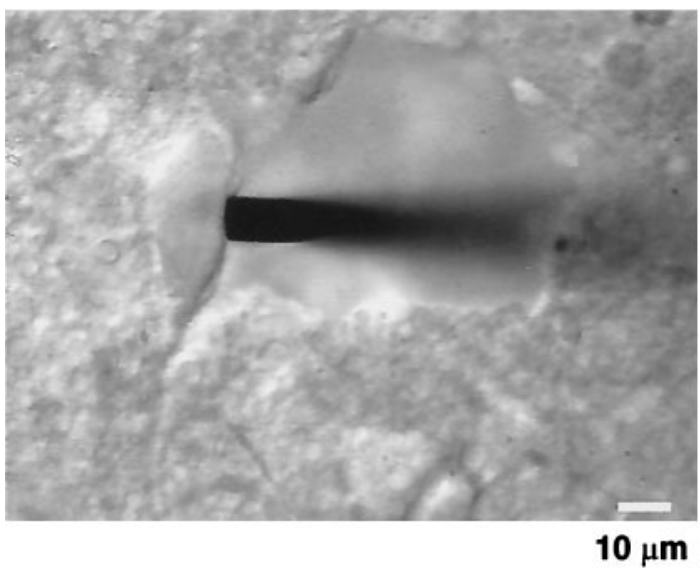

B

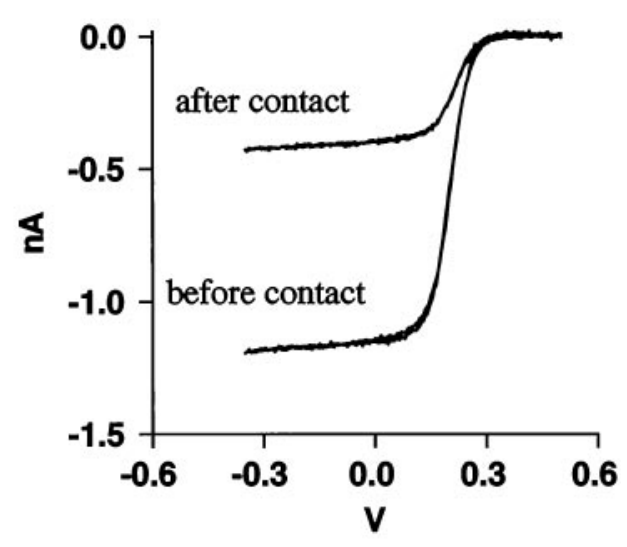

Figure 1. Cleaning procedure for amperometric recording in substantia nigra. A, Appearance of substantia nigra neuron during amperometric recording. A substantia nigra neuron from the pars compacta was partly freed from overlying connective tissue with the help of a saline-filled glass pipette. A carbon fiber electrode (EDP-insulated type) then was positioned to attain contact with the exposed part of the neuronal soma. Scale bar, $10 \mu \mathrm{m}$. $B$, Cyclic voltammograms with a scan rate of $100 \mathrm{mV} / \mathrm{sec}$ were performed in potassium ferricyanide solution (see Materials and Methods). The vertical excursion gives the limiting current, an indication of the sensitivity of the electrode. The limiting current is reduced by a factor of $\sim 5$ after contact with brain tissue.

\section{Response to local glutamate and $\mathrm{K}^{+}$stimulations}

Once the background activity was determined, the preparation usually was stimulated in an attempt to modify the pattern of the amperometric events. Figure $3 A$ illustrates typical examples of events recorded before and during a 30 -sec-long local application of $0.3 \mathrm{~mm}$ glutamate. The frequency of amperometric events, which was $\sim 6 /$ min before the application, rose to $32 /$ min during and after the application, a fivefold increase (Fig. 3B). In several instances, repeated responses to multiple glutamate applications (up to four) could be elicited from the same cell. However, the responsiveness to glutamate and the resting frequency slowly decreased during the recording (Fig. $3 B, C$ ).

In total, 19 cells were stimulated with $0.3 \mathrm{~mm}$ glutamate under the conditions of Figure 3. For each experiment the event numbers were determined for control periods, before and between stimulations, as well as for test periods during and shortly after glutamate applications. The mean frequency obtained when all experiments were pooled together rose from 2.1 events/min during resting periods to 7.2 events/min during stimulations. The difference between these two values was significant, showing that amperometric events were sensitive to glutamate applications (Table 1). In individual experiments it was not always clear whether glutamate was excitatory because of the small total number of events that sometimes was obtained (occasionally 0 ). Of 14 cells in which this total number was five or larger, 11 showed a clear increase in frequency (test/control frequency ratios $\geq 2$ ). Thus it can be concluded that at least 11 of 19 equaling $58 \%$ of the cells responded to glutamate. In addition to the increase in event frequency illustrated in Figure 3, glutamate applications often led to a gradual and reversible increase in the background current (data not shown). This effect was attributed to the cumulative increase in the concentration of the released substance after liberation from distant sites located either in the recorded cell or in its neighbors.

To reveal the kinetics of the effects of glutamate application (Fig. $3 C$ ), we aligned responses to 10 glutamate stimulations (0.3 $\mathrm{mm}$ ) from five particularly responsive cells at the start of the applications, which ranged from 27 to $47 \mathrm{sec}$ in duration. In these

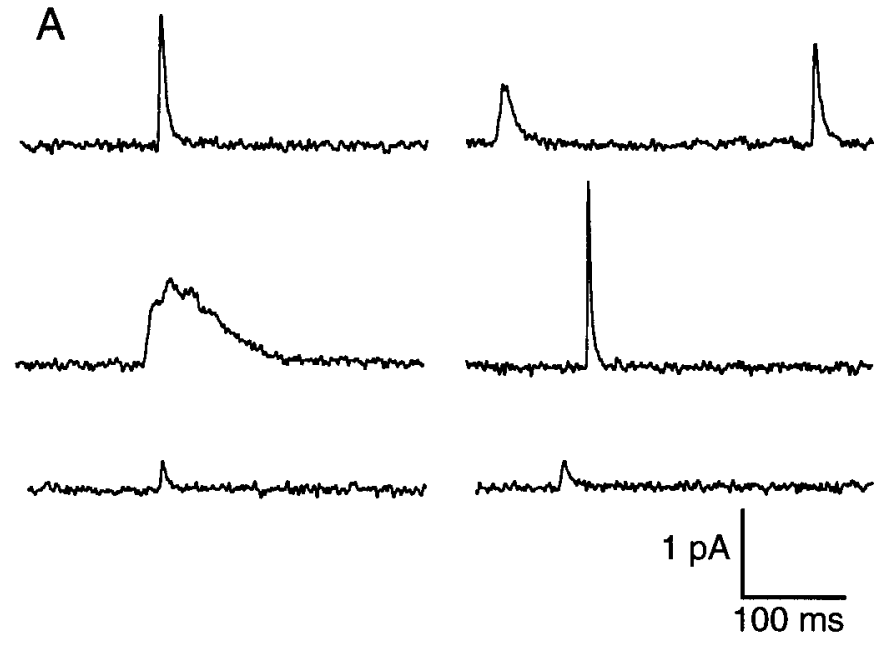

B

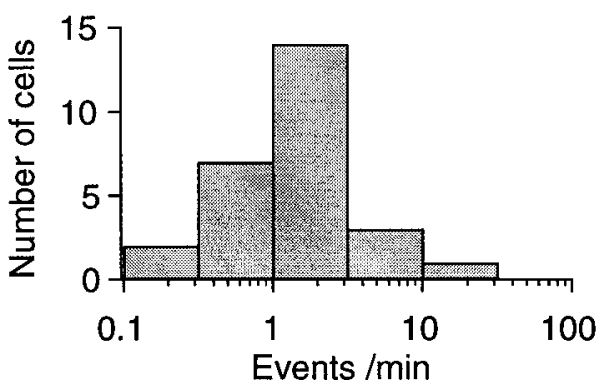

Figure 2. Spontaneous amperometric events recorded from a substantia nigra neuron. $A$, Examples are shown of spontaneous events recorded from a single cell. Notice the large variability in size and time course of these events. $B$, Shown is the distribution of the mean frequencies of spontaneous amperometric events in the different cells in which events could be recorded. 
A
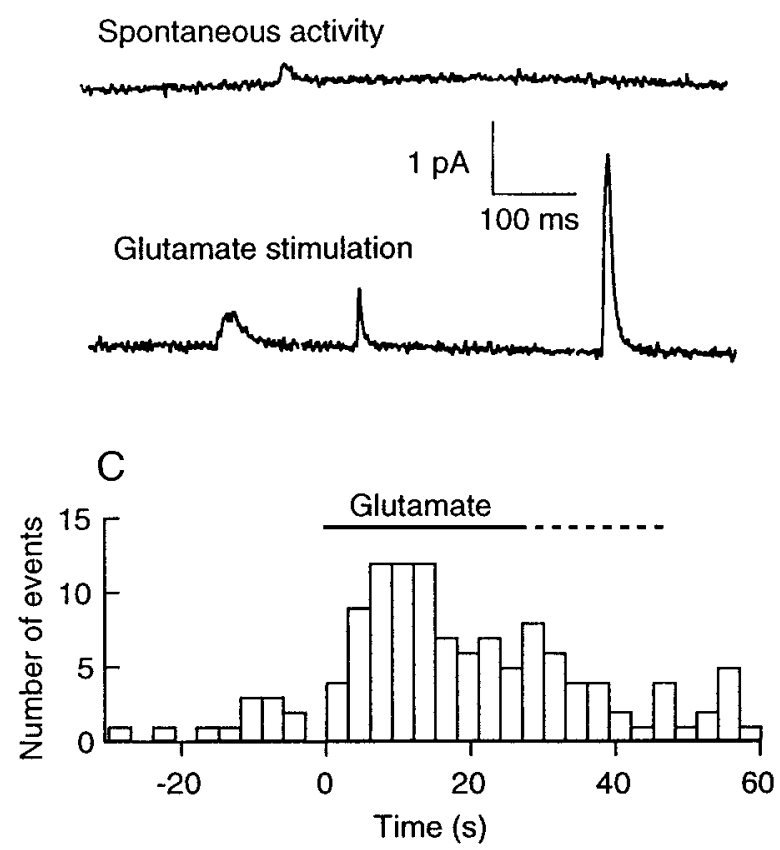

B
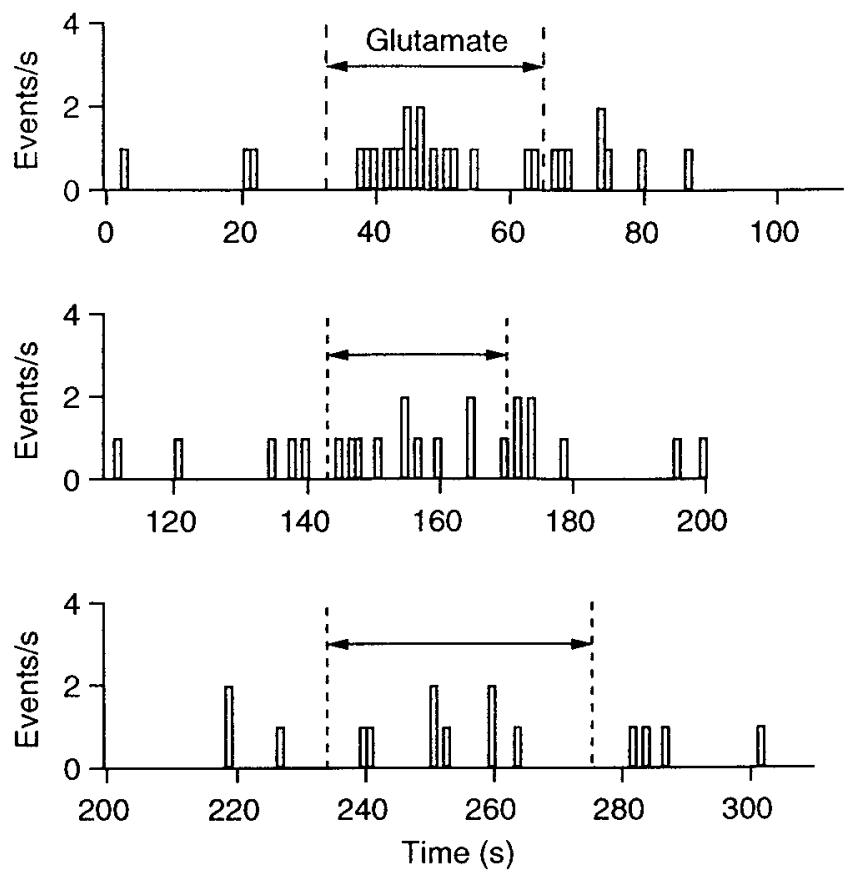

Figure 3. Enhancement of the frequency of amperometric events by glutamate applications. $A$, Sample recordings at rest (top) and a few seconds after local application of $0.3 \mathrm{~mm}$ glutamate (bottom). $B$, Timing of amperometric events after three successive applications of glutamate to the same cell. The three plots are consecutive. The events illustrated in $A$ are taken before and during the first glutamate application to this cell. Each event is represented by a vertical box. There are 17 events during the first application, which lasted $30 \mathrm{sec}$, as compared with three events during the preceding $30 \mathrm{sec}$ period. Note that the effects of glutamate become weaker for the second and third applications than for the first. $C$, Effect of glutamate on event frequency. Results are cumulative from 10 applications obtained in five cells. The results have been aligned with respect to the start of the applications. Applications ranged between $27 \mathrm{sec}$ (solid line) and $47 \mathrm{sec}$ (the end of the dotted line) in duration.

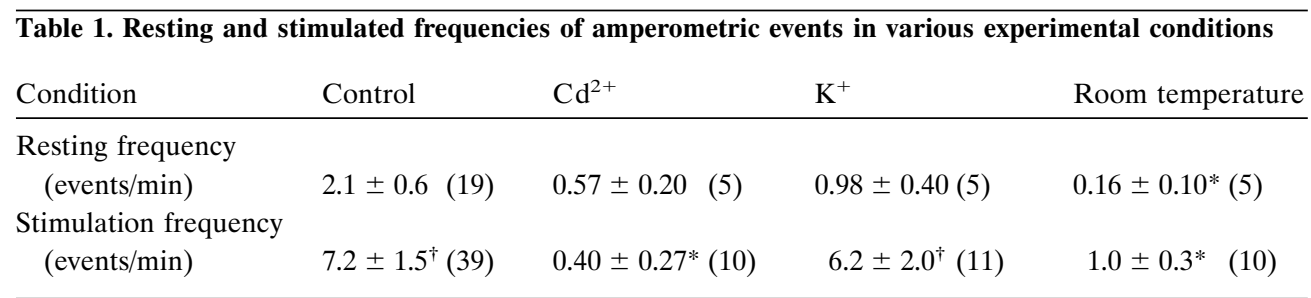

For each cell, amperometric events were counted during resting and stimulated periods. A typical sequence comprised 2 min of rest, one stimulation of $30 \mathrm{sec}$, a rest of $1.5 \mathrm{~min}$, a second stimulation of $30 \mathrm{sec}$, and a final rest of $1.5 \mathrm{~min}$. Control experiments refer to glutamate $(300 \mu \mathrm{M})$ stimulation performed in normal saline at $32-35^{\circ} \mathrm{C}$. $\mathrm{Cd}^{2+}$ experiments were performed in a saline supplemented with $50 \mu \mathrm{M} \mathrm{Cd^{2+ }}$. $\mathrm{K}^{+}$experiments were performed with $100 \mathrm{~mm} \mathrm{~K}^{+}$in the stimulation solution instead of glutamate. First row, Resting frequencies for each cell; numbers of cells in each condition are given in parentheses. Second row, Stimulated frequencies for each stimulation; total numbers of stimulations in each condition are given in parentheses. Values are means $\pm \mathrm{sem}$; *significantly different from control; $t$ test; $p<0.05$. ${ }^{\dagger}$, Significantly different from resting frequency; paired $t$ test; $p<0.05$. For each stimulation the stimulated frequency was compared with the resting frequency corresponding to the periods before and after this stimulation.

cells the glutamate applications increased the event frequency on average by 10 -fold, from 2.4 events/min to 24 events/min. The peak of the response was attained $9 \mathrm{sec}$ after the start of the stimulus. Over the next $20 \mathrm{sec}$ of maintained stimulation, the response subsided by approximately twofold. On cessation of the stimulation the frequency returned to control values with a time constant of $6.2 \mathrm{sec}$ (determined by aligning the responses with the offset of the applications). The rather slow kinetics of onset and of recovery of the glutamate-induced stimulation may reflect the slow diffusion of glutamate through the slice tissue.

In another series of experiments the cells were stimulated locally by using puffer pipettes loaded with a solution containing $100 \mathrm{~mm} \mathrm{~K}^{+}$. The high $\mathrm{K}^{+}$stimulations led to a strong increase in the frequency of amperometric events, similar to that obtained with glutamate (see Table 1).

\section{Event characteristics at rest and after glutamate stimulation}

Histograms of half-widths and charge integrals of the individual events are shown in Figure $4, A$ and $B$. The modal values of the half-width and the charge integral were $3.5 \mathrm{msec}$ and $4.5 \mathrm{fC}$, respectively. As illustrated in Figure $4 C$, there was no significant difference in the charge integral distribution of spontaneously occurring events and glutamate-evoked events (KolmogorovSmirnov test). 
A

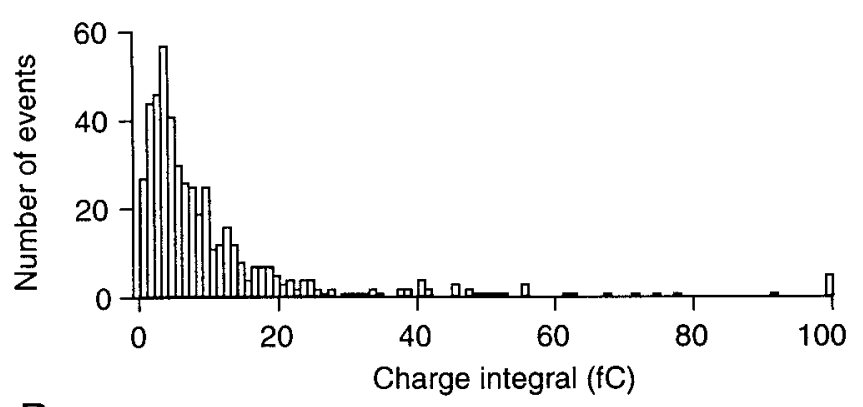

B

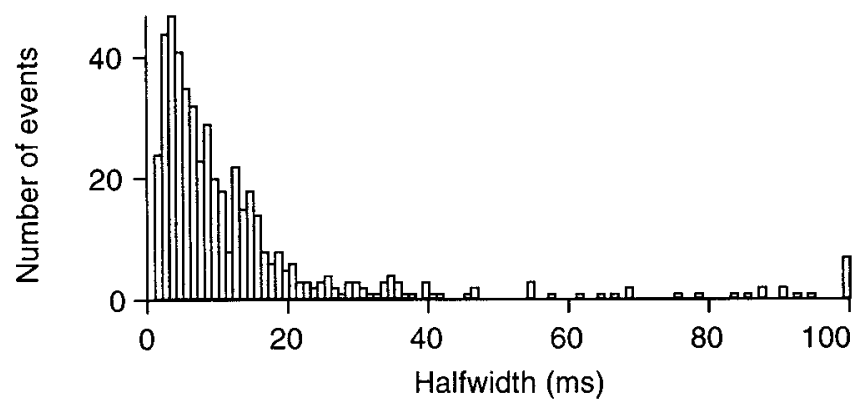

C

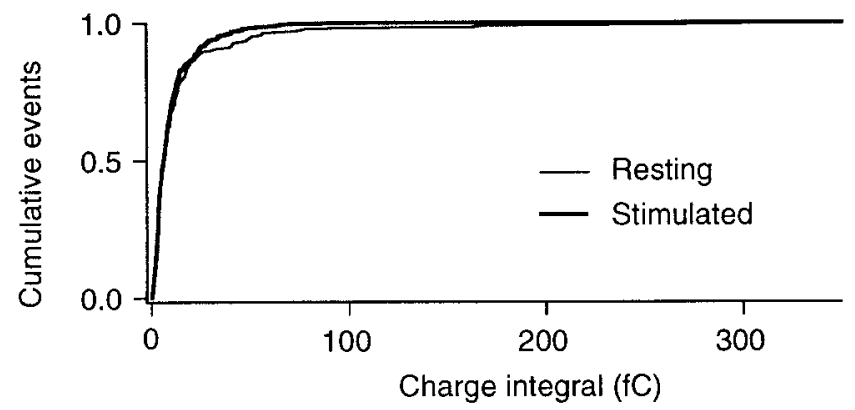

Figure 4. Summary histograms of amperometric event characteristics (498 events). $A$, Charge integral. $B$, Half-widths (with $200 \mathrm{~Hz}$ of low-pass filtering). $C$, Comparison of cumulative charge histograms for spontaneous and for stimulated events. Both histograms have been normalized. There was no significant difference between the two curves.

\section{The response to glutamate depends on voltage- dependent $\mathrm{Ca}^{2+}$ entry}

Bulk release of dopamine in the substantia nigra can be blocked by manipulations that block voltage-dependent $\mathrm{Ca}^{2+}$ entry into neurons (Geffen et al., 1976; Cheramy et al., 1981; Rice et al., 1994). Likewise, it seemed plausible that glutamate and $\mathrm{K}^{+}$ stimulations increased the frequency of amperometric events by promoting voltage-dependent $\mathrm{Ca}^{2+}$ entry into substantia nigra neurons. We therefore examined whether glutamate-induced stimulation would be sensitive to the inorganic $\mathrm{Ca}^{2+}$ channel blocker, cadmium $\left(\mathrm{Cd}^{2+}\right)$. In the presence of $\mathrm{Cd}^{2+}(50 \mu \mathrm{M})$ the frequency of spontaneous events was $0.57 \mathrm{event} / \mathrm{min}$, smaller than in normal solution (2.1 events/min). Although the difference was not significant at the $p<0.05$ level, this result suggests that blockage of $\mathrm{Ca}^{2+}$ channels leads to a decrease of the background frequency of amperometric events. The mean event frequency during and shortly after stimulation in $\mathrm{Cd}^{2+}$ was 0.40 event/min, significantly different from the value obtained in control stimula- tion (see Table 1). This result shows that $\mathrm{Cd}^{2+}$ effectively blocks the responsiveness of amperometric events to glutamate. Indeed, there was no indication of an enhancement of event frequency by glutamate in $\mathrm{Cd}^{2+}$-containing saline. The lack of responsiveness to glutamate could not be attributed to the preparations, because the results of three cells interleaved in the $\mathrm{Cd}^{2+}$ experiments gave resting and stimulated frequencies of 1.4 and 5.3 events $/ \mathrm{min}$, close to the overall average, and in each cell a clear response to glutamate was apparent.

\section{Influence of temperature on spontaneous and evoked amperometric events}

Ordinarily, recordings were performed at $32-35^{\circ} \mathrm{C}$. However, a series of experiments was performed at $21-22^{\circ} \mathrm{C}$ to investigate the influence of temperature in resting and stimulated conditions. The mean frequency of amperometric events was only 0.16 event / min in unstimulated cells at $21-22^{\circ} \mathrm{C}, 13$ times lower than at $32-35^{\circ} \mathrm{C}$ (see Table 1). Likewise, in the presence of glutamate the event frequency was significantly lower than in control experiments. After stimulation by glutamate the frequency rose to 1.0 event/min; however, because of the low frequency of events, the difference to the resting frequency did not reach statistical significance. Control experiments at the usual temperature were interleaved between the low-temperature experiments and gave results close to the overall mean. These results indicate that performing experiments at room temperature drastically reduces the resting and stimulated frequency of amperometric events.

\section{DISCUSSION}

The present work demonstrates that discrete amperometric signals can be recorded at the somata of substantia nigra neurons in brain slices. Two experimental manipulations were found to be essential for the successful recording of such events: the cell had to be cleared of surrounding tissue, and the preparation had to be warmed above $30^{\circ} \mathrm{C}$. These procedures should be applicable to other brain regions.

In previous studies of cultured cells, such as adrenal chromaffin cells, unitary amperometric events have been attributed to vesicular transmitter release (Wightman et al., 1991; Urena et al., 1994; Chen et al., 1995; Zhou and Misler, 1995; Chow et al., 1996). In our experiments, several properties of the amperometric events argue strongly for an exocytotic mechanism and against a carrier mechanism. First, some events reached an amplitude of $20 \mathrm{pA}$, corresponding to a release rate of at least $6 \times 10^{7}$ molecules $/ \mathrm{sec}$. Single transmembrane transporters are unlikely to function at such a high rate. Second, the pulsatile nature of the signals would require the abrupt submillisecond or millisecond turning on and off of the transporter-uncharacteristic behavior for carriers. Third, there is no obvious mechanism by which glutamate could activate such a carrier. Finally, the earlier report that GBR 12909, a selective dopamine uptake inhibitor, increases levels of dopamine in the extracellular fluid strongly argues against a primary role of (reversed) uptake in release of dopamine (Cragg et al., 1997). Rather, it appears that the primary role of the transporter is to terminate dopamine action by the removal of the transmitter from the extracellular fluid. We therefore conclude that the amperometric events correspond to exocytosis.

We have no direct evidence about the nature of the oxidizable substance that was detected. However, because dopamine is the major catecholamine neurotransmitter that is known to be contained in, and released from, substantia nigra neurons (Rice et al., 1994; Cragg et al., 1997), it is most likely that the amperometric 
events represent released dopamine. In the pars compacta of the substantia nigra, the proportion of dopamine-containing cells was estimated at $85 \%$ by Yung and colleagues (1991), in reasonably good agreement with the proportion of cells in the present study that displayed amperometric events (93\%). It would be valuable in future experiments to examine how inhibitors of the reuptake mechanism for dopamine (and other catecholamines) affect the size and time course of the individual amperometric events.

Zhou and Misler (1995) recorded amperometric events from the somata of cultured neurons from superior cervical ganglia and attributed the signals to presynaptic release from overlying nerve terminals rather than to somatic release. We do not believe that the signals we recorded in the substantia nigra originated from presynaptic nerve terminals; whereas projections of serotoninand noradrenalin-containing neurons have been described in the substantia nigra, the density of these projections is very low, and, furthermore, most projections lead to synapses with dendrites in the pars reticulata rather than with pars compacta somata (Nedergaard et al., 1988) (for review, see Lacey, 1993). In addition, it is unlikely that the few catecholamine- or indolamine-releasing nerve terminals would have survived the extensive "cleaning" procedure that preceded recording. Thus, our recording of amperometric events from $>90 \%$ of the cells we studied is difficult to reconcile with the idea that the signals arise from serotonergic or noradrenergic synaptic terminals. We therefore argue that the signals were coming from the somata of the recorded cells. Consistent with this proposal, somatic exocytosis of dopamine and of other neurotransmitters has been reported in molluscan neurons and in peripheral neurons in culture (Dan et al., 1994; Chen et al., 1995; Huang and Neher, 1996).

The charge distribution histogram for amperometric events has a peak at $4.5 \mathrm{fC}$ and a long tail component that may indicate a separate vesicle population with a much larger content; however, the frequency of larger events was too low to allow for the confirmation of this idea. The peak value of the charge histogram corresponds to $2.8 \times 10^{4}$ elementary charges. Because dopamine has two redox sites susceptible to oxidation, the corresponding number of dopamine molecules is 14,000 . Charge distributions previously obtained for dopamine release from somata of Planorbis neurons are biphasic, with a small and a large vesicle population containing $\sim 40,000$ and 800,000 molecules per vesicle, respectively (Chen et al., 1995). Likewise, serotonin release from leech neurons revealed two vesicle populations with contents near 5000 and 80,000 molecules per vesicle, respectively (Bruns and Jahn, 1995). In the latter study it was suggested that the small and large quantal sizes originate from small clear vesicles and from large electron-dense vesicles, respectively. On the basis of this earlier work, it appears plausible that the main population of quanta in the present work also corresponds to small, clear vesicles, whereas the tail in the distribution may be attributable to larger, electron-dense vesicles.

In the present study, applications of glutamate or $\mathrm{K}^{+}$enhanced the frequency of amperometric events. Earlier bulk release studies showed that $\mathrm{K}^{+}$-evoked dopamine secretion from the substantia nigra depends on the presence of extracellular $\mathrm{Ca}^{2+}$ ions (Geffen et al., 1976; Cheramy et al., 1981; Rice et al., 1994). Likewise, the present finding that $\mathrm{Cd}^{2+}$ blocks the response to glutamate indicates that the intracellular $\mathrm{Ca}^{2+}$ concentration tightly controls the event frequency. The notion of extrasynaptic $\mathrm{Ca}^{2+}$-dependent exocytosis is in accord with recent studies show- ing that $\mathrm{Ca}^{2+}$ concentration elevation enhances vesicular release from the somata of isolated snail neurons and of mammalian neurons in culture (Dan et al., 1994; Chen et al., 1995; Huang and Neher, 1996).

\section{REFERENCES}

Björklund A, Lindvall O (1975) Dopamine in the dendrites of substantia nigra neurons: suggestions for a role in dendritic terminals. Brain Res 83:531-537.

Bruns D, Jahn R (1995) Real-time measurement of transmitter release from single synaptic vesicles. Nature 377:62-65.

Chen G, Gavin PF, Luo G, Ewing AG (1995) Observation and quantitation of exocytosis from the cell body of a fully developed neuron in Planorbis corneus. J Neurosci 15:7747-7755.

Cheramy A, Leviel V, Glowinski J (1981) Dendritic release of dopamine in the substantia nigra. Nature 289:537-542.

Chow RH, Klingauf J, Heinemann C, Zucker RS, Neher E (1996) Mechanisms determining the time course of secretion in neuroendocrine cells. Neuron 16:369-376.

Cragg SJ, Rice ME, Greenfield SA (1997) Heterogeneity of electrically evoked dopamine release and reuptake in substantia nigra, ventral tegmental area, and striatum. J Neurophysiol 77:863-873.

Dan Y, Song H-J, Poo M-M (1994) Evoked neuronal secretion of false transmitters. Neuron 13:909-917.

Edwards FA, Konnerth A, Sakmann B, Takahashi T (1989) A thin slice preparation for patch-clamp recordings from neurons of the mammalian central nervous system. Pflügers Arch 414:600-612.

Geffen LB, Jessell TM, Cuello AC, Iversen LL (1976) Release of dopamine from dendrites in rat substantia nigra. Nature 260:258-260.

Huang L-YM, Neher E (1996) $\mathrm{Ca}^{2+}$-dependent exocytosis in the somata of dorsal root ganglion neurons. Neuron 17:135-145.

Lacey MG (1993) Neurotransmitter receptors and ionic conductances regulated in the activity of neurons in substantia nigra pars compacta and ventral tegmental area. Prog Brain Res 99:251-276.

Mercer L, del Fiacco M, Cuello AC (1979) The smooth endoplasmic reticulum as a possible storage site for dendritic dopamine in substantia nigra neurones. Experientia 35:101-103.

Nedergaard S, Bolam JP, Greenfield SA (1988) Facilitation of a dendritic calcium conductance by 5 -hydroxytryptamine in the substantia nigra. Nature 333:174-177.

Nirenberg M, Chan J, Liu Y, Edwards RH, Pickel VM (1996) Ultrastructural localization of the vesicular monoamine transporter-2 in midbrain dopaminergic neurons: potential sites for somatodendritic storage and release of dopamine. J Neurosci 16:4135-4145.

Rice ME, Richards CD, Nedergaard S, Hounsgaard J, Nicholson C, Greenfield SA (1994) Direct monitoring of dopamine and 5-HT release in substantia nigra and ventral tegmental area in vitro. Exp Brain Res 100:395-406.

Schulte A, Chow RH (1996) A simple method for insulating carbon fiber microelectrodes using anodic electrophoretic deposition of paint. Anal Chem 68:3054-3058.

Urena J, Fernandez-Chacon R, Benot AR, Alvarez de Toledo GA, Lopez-Barneo J (1994) Hypoxia induces voltage-dependent $\mathrm{Ca}^{2+}$ entry and quantal dopamine secretion in carotid body glomus cells. Proc Natl Acad Sci USA 91:10208-10211.

Wassef M, Berod A, Sotelo C (1981) Dopaminergic dendrites in the pars reticulata of the rat substantia nigra and their striatal input. Combined immunocytochemical localization of tyrosine hydroxylase and anterograde degeneration. Neuroscience 11:2125-2139.

Wightman RM, Jankowski JA, Kennedy RT, Kawagoe KT, Schroeder TJ, Leszczyszyn DJ, Near JA, Dilberto Jr EJ, Viveros OH (1991) Temporally resolved catecholamine spikes correspond to single vesicle release from individual chromaffin cells. Proc Natl Acad Sci USA 88:10754-10758.

Yung WH, Häusser MA, Jack JJB (1991) Electrophysiology of dopaminergic and non-dopaminergic neurons of the guinea-pig substantia nigra pars compacta in vitro. J Physiol (Lond) 436:643-667.

Zhou Z, Misler S (1995) Amperometric detection of stimulus-induced quantal release of catecholamines from cultured superior cervicalganglion neurons. Proc Natl Acad Sci USA 92:6938-6942. 